\title{
Urban Spaces in Residential Areas as an Environment for Continuous Prevention and Rehabilitation - Design and Benefits
}

\author{
Barbara Skalna, Patrycja Haupt
}

\begin{abstract}
Facing the demographic changes is our common responsibility as researchers of all scientific disciplines. The forecasts based on statistical data show that in many countries in the nearest future the highest growth in population will be in the segment of 65-year-olds and older people. With age, mobility, intellectual ability and spatial orientation are reduced. Creating a proper living environment and public spaces to conduct prevention and rehabilitation training would have a positive impact on shaping and maintaining the quality of life of residents. The purpose of this multidisciplinary research was to create a model training for people with motor dysfunctions together with an optimal design of environment to conduct it on daily basis. Currently, a great emphasis in the design of urban areas is placed on the physical and social activity of children and youth (playgrounds, skate parks, sports equipment), as well as people with a high degree of physical fitness (sports fields and equipment, outdoor gyms, running paths, nordic walking, cross-country skiing). The aim of the work was to explore the possibilities and prove effectiveness of basic functional training with sensory elements in non-standard activity spaces intended for people with reduced low physical activity and motor disorders, not requiring additional devices, using elements of nature, such as terrain, water, greenery - as well as elements of small architecture.
\end{abstract}

Keywords : Demographic changes, forecasts, population, emphasis, urban spaces.

\section{INTRODUCTION}

Facing the demographic changes should be common responsibility of the researchers of all scientific disciplines. The complexity of the problem needs to be addressed by multidisciplinary teams to come up with innovative complex solutions addressing current issues. The available forecasts based on statistical data show that in many countries the nearest future will be demographically characterized by the highest population growth in the segment of 65-year-olds and older people. At the end of 2014, the population of Poland was 38.5 million, of which over 8.5 million were people aged 60 and over (over 22\%) which proves the necessity of searching for solutions that would help to maintain fitness level and prevent dependency.

With age, mobility and intellectual ability as well as spatial orientation are reduced (over $70 \%$ of people with disabilities are those who have reached the age of 50). The main diseases occurring in the group of biologically disabled people are

Revised Manuscript Received on July 22, 2019.

Barbara Skalna, Academy of Physical Education in Cracow, Cracow University of Technology

Patrycja Haupt, Academy of Physical Education in Cracow, Cracow University of Technology injuries and diseases of the musculoskeletal system (in 54\%), the circulatory system (in 44\%), eye diseases (in 32\%) and neurological diseases (in 30\%). Creating a proper living environment with public urban spaces that would serve as everyday preventive training areas would have a positive impact on shaping and maintaining the efficiency and quality of life of residents.

\section{GENESIS}

According to the World Health Organization, the definition of quality of life determined by the state of health refers the areas of physical, mental, social performance included in the subjective self-assessment. The concept of quality of life is complex and can be addressed from many different perspectives. Physical activity, which is one of the determinants of lifestyle, is important in the physical and mental area. As a result of technological progress, man reduced his physical activity, resulting in lowering the body's efficiency. Therefore, it is particularly important for older people to maintain a good psychophysical condition by using appropriate physical activity, which is permanently included in the lifestyle of a person. Systematic training is an essential part of a lifestyle that determines health.

One of the possible training facilities that has a potential of implementation in the residential environment is the moto-sensory path. It was designed as a compact urban space - dedicated for permanent training - that is intended to serve people in the seniors' age and people who suffer from mobility restrictions as a result of illness or injury. It consists of various training and sensory posts is to help maintain or increase the mobility of beneficiaries. In addition to motor aspects, elements referring to nature - vegetation, presence of water, diverse surface, etc., are to affect the senses through smell, eyesight, sound and tactile sensations, introducing the user into a state of relaxation. The motosensory path is designed to familiarize people who, due to the reasons of reduced efficiency, experience fears and cease to use public spaces of the city - with the urban space and encourage them to fully benefit from living in the city. The home location is supposed to encourage users to endure external conditions in the immediate neighborhood, in protected well-known environment, in order to transfer positive experiences to more distant regions, taking full advantage of the cultural, educational and recreational offer of the city.

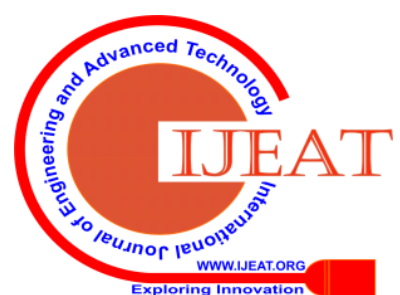




\section{OBJECTIVE OF RESEARCH}

Physical activity, which is one of the determinants of lifestyle, is important in the physical and mental health. Statistics show that the level of physical activity decreases with age and reduces efficiency of the musculoskeletal system. Older people become weaker and slower, which in turn increases the risk of falls and may lead to the development of motor disability. That is why it is so important to maintain good psychophysical condition by keeping an active lifestyle [1,2]. The systematic functional and preventive training seems to be a great idea of that. Motosensory functional training in urban space is dedicated mainly to seniors and people suffering from physical limitations as a result of a disease such as stroke, Parkinson's disease, multiple sclerosis. The proposal of training solutions is intended to help maintain or increase the mobility of beneficiaries. This solution reduces the costs of the public health sector and ensures the continuity of the rehabilitation process. The training is designed to familiarize with the urban space people who, due to the reasons of reduced mobility, experience fears do not use public spaces of the city. The home location is supposed to encourage users with open space in the immediate environment, in order to transfer positive experiences to taking full advantage of the cultural, educational and recreational offer of the city.

The purpose of the research is to specify methods of preventive functional training and the possibilities for designing the recreational space in residential areas and public spaces to serve these activities. Such activity can be progressed supervised by physiotherapist or alone with help of Android application or using graphic manuals. Currently the process of rehabilitation is mostly restricted to hospital areas and there are no facilities where the individual training can be conducted. A great emphasis in the design of urban areas is placed on the physical and social activity of children and youth (playgrounds, skate parks, sports equipment), as well as people with a high degree of fitness (sports fields and equipment, outdoor gyms, running paths, nordic walking, cross-country skiing). The aim of the research is to present an individual training in a non-standard activity spaces intended for people with reduced efficiency. The design of such areas would not require additional devices, nonetheless it would involve elements of nature, such as terrain, water, greenery as well as elements of small architecture.

The therapeutic purpose is optimal functioning in order to achieve the goal determined by the task, in selected environmental conditions. This will improve everyday activities, improves movement strategies dependent on musculoskeletal and nervous systems and in effect increase independence of beneficiaries. Creation of therapeutic space also has an impact on senso-motor integration - a process in which the human nervous system receives information from receptors by means of all the senses (touch, vestibular system receiving movement, body sensation i.e. proprioception, smell, sight, hearing, taste), then organizes and interprets them [3]. One of the main goals of the training is also to obtain/improve posture control. It is a dynamic ability to maintain and regain balance on a stable and unstable base. It improves the ability to maintain static and dynamic balance.
The planned activities improve the body's position in space, which is particularly disturbed in people after stroke [4]. The proper functioning of the nervous system depends on physical activity. Scientific research indicates that exercise leads to an increase in the number of neuronal connections in the brain by increasing and improving the transport of oxygen to the cells [3]. The training should improve cognitive functions and improve well-being. The purpose of the moto-sensory training is not only to maintain physical fitness at an appropriate level, but also to delay the need to depend on the help of other people. Aging is one of the risk factors for disability. In the social aspect, seniors undertaking physical activity are more independent and feel the improvement of the quality of life. Residing in a given space that stimulates the senses in a controlled manner, combined with appropriate motor training, can beneficially impact the motivation of users, as well as the obtained results [5].

\section{BENEFICIARIES}

Motosensory functional training in urban space is dedicated mainly to seniors and people suffering from physical limitations as a result of a disease such as stroke, Parkinson's disease, multiple sclerosis. People with physical disabilities may experience fear of situations that require moving in urban space - using public transport, pedestrian crossing. Movement disorders also cause the loss of independence in everyday activities, such as shopping. These people limit their activity to their place of residence and often give up their participation in social and cultural life. Space like this is suitable for people who need a continuous rehabilitation process and also do not have money for rehabilitation in special centers with a physiotherapist. A separate problem is a long term waiting for rehabilitation services in public hospitals and outpatient clinics. This often results in the loss of previously developed motor skills. Sensory elements have a therapeutic effect on people after stroke and other neurological diseases. In many cases, there is a problem with body sensation. Additionally sensory stimulation causes the brain plasticity process and influences the functional improvement of the motor system of these people [3,6]. Seniors are also beneficiaries. Their physical and functional fitness decreases with age. Their quality of life is reduced. Older people often have diseases that also require therapeutic movement - diabetes, degeneration of the joint [7]. In the same time therapeutic urban space can be the site of attractive walks for able persons and children. Through communal care for its aesthetics and condition, it can become a site that improves family relations, and can also be conducive to social contact. It can become an area for the exchange of experiences between dependent persons and their caretakers. 


\section{KINESIOTHERAPY AND SENSORY STIMULATION COMBINED}

The combination of kinesiotherapy (motion therapy) and sensory stimulation can be called moto-sensory therapy. Various activities are performed that stimulate the nervous system and help to integrate stimuli reaching the body. Numerous materials are used due to their sensory value e.g. rough, smooth, cold, warm. Training in urban space, which will be properly managed, can have a therapeutic effect on the movement system - balance exercises on an unstable, changing ground, exercise the gait function on a designated path, sitting on a bench, reaching for an object, going up the stairs. Aromatic plants or water elements will stimulate sensory and relaxing effects on the user of such space. The location in the backyard is therapeutic for people with hypersensitivity to noise. This is especially important for stroke patients who have great difficulty in focusing on the task being performed.

Under such conditions, postural control training is possible - important for balance. In addition, it allows to perform a movement task in a coordinated and economical way. Postural control is weakened by disorders related to the processing of sensory information. The combination of kinesiotherapy and sensory stimulation positively affects eccentric muscle control. It is needed to control the speed of movement (sitting on a chair, going down stairs) which is especially important for neurological patients [10].

Spasticity is known as a serious problem in people with central nervous system damage like stroke. It is defined as excessive resistance to muscle stretching. Repetition of strength and functional training may lead to a reduction in spasticity through improved neuromuscular coordination [5]. Less spasticity means greater freedom of movement, precision and speed. Sensory stimulation can be part of such therapy.

Physical fitness decreases with age. Aging is a physiological process associated with structural changes and activities: reduction of lean body mass, decrease in strength, flexibility of muscles, tendons and ligaments. In addition, aerobic efficiency is significantly reduced in the elderly. This results in balance disorders and increased risk of falls. It may lead to disability and dependence on others. Recent research shows that it is possible to increase muscle mass and strength under the influence of strength training in older people - even at a very advanced age. The combination of kinesiotherapy and sensory stimulation in the elderly influences such motor features as flexibility, balance and motor coordination. The use of such training affects the improvement of the quality of walking. Equivalent exercises are important in the prevention of falls. Stretching exercises, which are also provided in such training, allow for greater mobility $[11,12]$.

The combination of moto-sensory training affects central stabilization, activates deep muscles. When performing the activity, deep stabilizing muscles are activated first. A stable center allows the movement of distal parts of the body - the movement is smooth, in full range, and the movement pattern is correct. This is important for seniors and neurological patients $[3,13]$.

\section{TRAINING METHOD}

The suggested training plan consists of 6 randomly selected exercises, drawn from 7 specific groups, after 5 minutes every. Qualifying tests are suggested - a simple equivalent exercise and gait training strips to determine the user level of the 3 available difficulty variants. Created training plan, for each level of difficulty, has one exercise from the group: gait training, stretching, stabilization, postural control, eye-hand coordination, balance. The whole training lasts about 60 minutes. Time contains short breaks between exercises. Walking training is often the main therapeutic goal. Its function is, as far as possible, to preserve or restore the optimal walking pattern, which will be ergonomic and allow you to move on short and long distances. Stretching - it is the "hygiene" of the muscular system, allows to maintain the range of mobility in the joints and increase the mobility of tissues. In the case of neurological patients and the elderly, stretching and mobilization with movement are effective. Stabilization - it determines mobility. It is directly related to postural control and balance It allows one to get the freedom of movement of distal parts of the body. It allows you to control your posture while your body is moving in space and maintaining balance. Postural control - is associated with stabilization. It performs similar functions, it also allows you to maintain, achieve or regain balance while changing the position of the body or performing physical activity. Eye-hand coordination - is important for everyday activities such as writing, reaching for small things, basic activities like dressing, washing. In the proposed exercises, verbal games were used that divert attention from physical exercise and increase the difficulty of the task. They also have a therapeutic effect on the coordination process. Balance - is associated with stabilization and postural control. It performs similar functions. We have a static balance (when we are standing or sitting) and a dynamic balance (the ability to recover and maintain the balance during activity). Sensory training (relaxation) - it allows you to relax the whole body, regulate the breath and work of the heart. Different sensory stimuli reach the senses. On the surface of the hands and feet we have a lot of nerve endings. They receive the most sensory impressions. Intense sensory experiences strengthen the transmission of impulses to the brain, integration of stimuli and improvement of neuronal processes $[8,9,10]$.

One of the training methods is training under the supervision of an instructor who presents the selected exercise in a correct way and presents its purpose. The coach may be a physiotherapist from a nearby rehabilitation center or a person employed by the local senior club i.e. When the user performs the exercise, the trainer (verbally or manually) corrects the motion being performed. His role is also motivating and appreciate the effort of the participants as well as preparing the users before undertaking independent training. Later on the trainer only supervises the participants and helps if necessary. Users should acquire skills that will enable self-training and self-confidence.

Another method of training is independent training based on multimedia instruction. A special application containing audiovisual materials has been created for this purpose. There is 
a short movie for each exercise (with a voice of the teacher and subtitles to choose from). There are 3 levels of difficulty. The first step in using the application is to perform 2 exercises - tests. Their result is the basis for qualifications to the appropriate level of difficulty. On this basis, a set of exercises is randomly selected. Training based on the application can replace the meeting with the instructor and allows you to take independent training in the created space.

There is also a possibility of training based on graphic instruction. Professional pictograms have been created for this purpose. Do each exercise assigned to one figure, which represents the starting position and the set movement. In addition, clear instruction (separate for each level of difficulty) below the picture. In this way, they will have a full graphical instruction, which will be placed on the board at each exercise stand. The same instruction is also available in the application. This type of training is recommended for more advanced participants who have learned the correct way of conducting exercises before.

\section{TESTING METHOD AND EVALUATION}

Methods of testing the effectiveness of training in urban space can be functional tests used in physiotherapeutic practice. They are objective research tools. In case they are done before and after a series of trainings, they can also be used as a tool to evaluate the training.

The following research tools are recommended: Index Barthel (Modified Scale Barthel) - evaluates the degree of independence can be used as a preliminary assessment of people starting workouts. If a person scores too few points on the scale, he or she will have difficulty performing exercises and the therapy will not be suitable for such person.

Test "get up and go" (TUG test - the timed "up and go" test) evaluates the possibility of transition from sitting to standing position, walking on short distance (equal to the average length of pedestrian crossing), turning back by 180 degrees and transition from position standing up to sitting. The task of the tested person is to complete the task as soon as possible. The test will be repeated twice, the time is measured with a stopwatch. Given the results of a better sample. The test contains a point scale and assesses the risk of falls.

POMA (Performance Oriented Mobility Assessment) / Tinetti Test - evaluates the balance when sitting, standing up, standing up, turning. It also evaluates gait: initiation, step length and height, step symmetry, gait continuity, gait path and body positions. Assessment of the balance and quality of walking gives information about the possible risk of falling of the tested person.

Clinical Test for Sensory Interaction In Balance (CTSIB) assessment of balance in various sensory conditions. Test for static balance assessment in six situations using vestibular and proprioceptive information. The test contains a point scale and assesses the risk of falls.

Functional Reach Test (FR) - the test gives information about the dynamic ability to maintain the balance in a standing position. Depending on the result, the interpretation indicates a double risk of falls or a fourfold increased risk of falls.

6-Minute Walk Test (6MWT) - a 6-minute walk test determines the distance a person can walk on a flat surface in 6 minutes. The walking path, according to standards, is $30 \mathrm{~m}$ long. People who use orthopedic help on a daily basis can also perform this test.

Most tests assess the balance because training in the open space has the greatest impact on it. This is due to unstable and changing ground. Balance is a very important factor in the prevention of falls [11].

\section{RESULTS AND CONCLUSION}

The result of the research was inventing a training method as well as designing urban space for conducting everyday training. Through appropriate shaping of publicly accessible urban spaces, also those in the immediate vicinity of the place of residence, the comfort of people with reduced mobility can be influenced. It is also possible to improve mobility and spatial orientation training by properly designing these spaces, in particular using natural elements. Terrain alternative communication routes and walking areas as a form of physical fitness training, especially in different atmospheric conditions, natural elements - greenery and water - as landmarks, by their multi-sensory perception, gardens urban farms, as a form of aromatherapy and a place of social integration at relatively low costs, positively influence the physical fitness of the population.

The factors that most liked and supported physical activity on the outside turned out to be visual stimuli, such as nature, the possibility of observing it and communing with it. In turn, natural auditory stimuli, such as birdsong, accompanying physical activity in the surroundings of the "fresh air" turned out to be important. The open space, which gives freedom of movement, was also important. The element of support is also air quality, because the beneficiaries valued the possibility of breathing fresh air.

The result of the research showed how carefully designed urban space can become a therapeutic space. The therapeutic purpose is optimal functioning in order to achieve the goal determined by the task, in selected environmental conditions. This will improve everyday activities, improves movement strategies dependent on musculoskeletal and nervous systems and in effect increase independence of beneficiaries. Creation of therapeutic space also has an impact on senso-motor integration.

In pilot studies increase in functional efficiency was observed in the field of basic everyday activities such as: balance in the seat, standing up, standing up, while turning and sitting. Inception, step length and height, step symmetry, gait continuity, gait path and body positions (Tinetti Test), which directly affects the improvement of the static and dynamic balance of the patients.

Based on a comparison of the results from before and after a series of pilot training sessions in all study groups, one can talk about the effectiveness of actions undertaken on the local rehabilitation path. It is advisable, however, to broaden the scope of research by increasing the study group and establishing a control group. The developed results of all the functional tests used in the assessment of the effectiveness of the moto-sensory path will be presented soon.

In conclusion - the research proved: 
- the legitimacy of implementation of non-standard spaces and forms of preventive training,

- the necessity of further multidisciplinary research on possible benefits,

- the need of searching for optimal methods of implementation and training in the residential neighborhood..

\section{REFERENCES}

1. G. 1. A. Ćwirlej -Sozańska, "Physical activity and the health condition of older people," Medical Review, vol. 2, pp.173-181, 2014.

2. T. Paillard, Y. Rolland and P. de Souto Barreto, "Protective Effects of Physical Exercise in Alzheimer's Disease and Parkinson's Disease: A Narrative Review," J. Clin. Neurol., vol. 11(3), pp. 212-219, July 2015.

3. A. Moghadasi, G. Ghasemi, E. Sadeghi-Demneh and M. Etemadifar, "The Effect of Total Body Resistance Exercise on Mobility, Proprioception and Muscle Strength of the Knee in People with Multiple Sclerosis," J Sport Rehabil., vol 24(1), pp. 1-22, January 2019.

4. $\quad$ N. Eckardt, "Lower-extremity resistance training on unstable surfaces improves proxies of muscle strength, power and balance in healthy older adults: a randomised control trial," BMC Geriatr., vol. 16(1), p. 191, November 2016

5. M. A. Dimyan and L. G. Cohen, "Neuroplasticity in the context of motor rehabilitation after stroke," Nat. Rev. Neurol., vol. 7(2), pp. 76-85, Februar 2011.

6. K. I. Erickson, A. G. Gildengers and M. A. Butters, "Physical activity and brain plasticity in late adulthood," Dialogues Clin Neurosci., vol. 15(1), pp. 99-108, March 2013.

7. A. Dunsky, T. Yahalom, M. Arnon and R. Lidor, "The use of step aerobics and the stability ball to improve balance and quality of life in community-dwelling older adults - a randomized exploratory study," Arch Gerontol Geriatr., vol. 71, pp. 66-74, July 2017.

8. C. S. Johnson and K. M. McLeod, "Relationship between Fear of Falling and Preceived Difficulty with Grocery Shopping," J Frailty Aging. Vol. 6(1), pp. 33-36, 2017

9. M. Bjerk, T. Brovold, D. A. Skelton, T. Liu-Ambrose and A. Bergland, "Effects of a falls prevention exercise programme on health-related quality of life in older home care recipients: a randomised controlled trial," unpublished.

10. V. A. Goodwin, S. H. Richards, W. Henley, P. Ewings, A. H. Taylor and J. L. Campbell, "An exercise intervention to prevent falls in people with Parkinson's disease: a pragmatic randomised controlled trial," J Neurol Neurosurg Psychiatry, vol. 82(11), pp. 1232-8, November 2011.

11. C. Sherrington, A. Tiedemann, N. Fairhall, J. Close and S. R. Lord, "Exercise to prevent falls in older adults: an updated meta-analysis and best practice recommendations," NSW Public Health Bulletin, vol. 22(4), pp. 8-83, June 2011.

12. B. F. Olij, V. Erasmus, L. M. Barmentloo, A. Burdorf, D. Smilde, Y. Schoon, van der Velde and S. Polinder, "Evaluation of Implementing a Home-Based Fall Prevention Program among Community-Dwelling Older Adults," Int J Environ Res Public Health., vol.16(6), p. 1079, March 2019

13. W. Bouaziz, P. O. Lang, E. Schmitt, G. Kaltenbach, B. Geny and T. Vogel, "Health benefits of multicomponent training programmes in seniors: a systematic review," Int J Clin Pract., vol. 70(7), pp. 520-36, July 2016

14. W. Bouaziz, T. Vogel, E. Schmitt, G. Kaltenbach, B. Geny and P. O. Lang, "Health benefits of aerobic training programs in adults aged 70 and over: a systematic review," Arch Gerontol Geriatr., vol. 69, pp. 110-127, March - April 2017.

15. T. M. Steffen, T. A. Hacker and A. Mollinger, "Age- and Gender-Related Test Performance in Community-Dwelling Elderly People: Six-Minute Walk Test, Berg Balance Scale, Timed Up \& Go Test, and Gait Speeds," Physical Therapy, vol. 82(2), pp. 128-137, February 2002.

16. S. Köpke and G. Meyer, "The Tinetti test - Babylon in the geriatric assessment," vol. 39(4), pp. 288-291, August 2006

17. J. C. Menant, D. Schoene, M. Sarofim and S. R. Lord, "Single and dual task tests of gait speed are equivalent in the prediction of falls in older people: a systematic review and meta-analysis," Ageing Res Rev., vol. 16, pp. 83-104, July 2014.

18. Ergonomia wobec procesu starzenia się społeczeństwa i kadry pracowniczej / ed.. Maciej Złowodzki, Tadeusz Juliszewski, Halszka
Ogińska, Anna Taczalska, Karolina Trzyniec ; Politechnika Krakowska im. Tadeusza Kościuszki. - Kraków : Wydaw. PK, 2018. - 329

19. Trojanowska, Monika. Parki i ogrody terapeutyczne. Red. . Wydawnictwo Naukowe PWN, 2017, 232 s. ISBN 978-83-01-19249-5 